\title{
Effectiveness of pneumococcal conjugate vaccine against presumed bacterial pneumonia hospitalisation in HIV-uninfected South African children: a case-control study
}

\author{
Shabir A Madhi, ${ }^{1,2,3}$ Michelle J Groome, ${ }^{1,2}$ Heather J Zar, ${ }^{4}$ Constant N Kapongo, ${ }^{5}$ \\ Christine Mulligan, ${ }^{4}$ Susan Nzenze, ${ }^{1,2}$ David P Moore, ${ }^{1,2}$ Elizabeth R Zell, ${ }^{6}$ \\ Cynthia G Whitney, ${ }^{6}$ Jennifer R Verani ${ }^{6}$
}

\begin{abstract}
- Additional material is published online only. To view please visit the journal online (http://dx.doi.org/10.1136/ thoraxjnl-2014-206593).

For numbered affiliations see end of article.
\end{abstract}

\section{Correspondence to}

Professor Shabir A Madhi, National Institute for Communicable Diseases 1 Modderfontein Road, Sandringham, Gauteng 2131, South Africa;

shabirm@nicd.ac.za

Received 19 November 2014 Revised 22 May 2015 Accepted 29 May 2015 Published Online First 19 June 2015

\section{SLinked}

- http://dx.doi.org/10.1136/ thoraxjnl-2015-207191

\section{CrossMark}

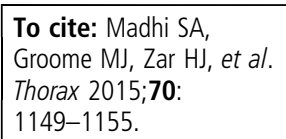

\section{ABSTRACT}

Introduction We evaluated pneumococcal conjugate vaccine (PCV) effectiveness against hospitalisation for presumed bacterial pneumonia (PBP) in HIV-uninfected South African children. 7-valent PCV was introduced in April 2009 using a $2+1$ schedule (doses at age 6, 14 and 39 weeks), superseded with 13-valent PCV in May 2011.

Methods A matched case-control study was conducted at three public hospitals (Soweto, Cape Town and KwaZulu-Natal) between April 2009 and August 2012. PBP cases had either WHO defined radiographically confirmed pneumonia or 'other infiltrate' on chest radiograph with C-reactive protein $\geq 40 \mathrm{mg} / \mathrm{L}$. Hospitalised controls were children admitted with a disease unlikely to be pneumococcal and matched for case age, site and HIV infection status. Age-matched community controls were enrolled from Soweto. Adjusted vaccine effectiveness (aVE) was estimated using conditional logistic regression.

Results Of 1444 HIV-uninfected enrolled PBP cases, 1326 had $\geq 1$ hospital controls ( $n=2075)$. Overall, aVE of an up-to-date PCV schedule was $20.1 \%$ (95\% $\mathrm{Cl}-9.3 \%$ to $41.6 \%)$ in children aged $\geq 8$ weeks and $39.2 \%(95 \% \mathrm{Cl} 8.46 \%$ to $59.6 \%)$ among children 16-103 weeks of age. There were 889 PBP cases in Soweto with hospital controls and $\geq 1$ community control $(n=2628)$. The aVE using community controls was similar compared with hospital controls in Soweto, including $32.1 \%(95 \% \mathrm{Cl} 4.6 \%$ to $51.6 \%)$ and $38.4 \%$ (95\% Cl $7.7 \%$ to $58.8 \%$ ), respectively, in age group $\geq 8$ weeks and $52.7 \%(95 \% \mathrm{Cl} 25.7 \%$ to $69.9 \%)$ and $53.8 \%(95 \% \mathrm{Cl} 19.5 \%$ to $73.5 \%)$, respectively, in age group 16-103 weeks.

Conclusions PCV implemented using a $2+1$ schedule in the routine infant immunisation programme was effective at preventing PBP in HIV-uninfected children. Effectiveness estimates were similar to efficacy measured by earlier randomised controlled trials using different vaccination schedules.

\section{INTRODUCTION}

Worldwide, pneumococcal disease is estimated to cause 450000 deaths in children age less than 5 years annually, $90 \%$ of which are due to non-

\section{Key messages}

What is the key question?

- This multicentred case-control study in South Africa evaluated the effectiveness of pneumococcal conjugate vaccine (PCV) in preventing pneumonia, following its introduction into the public immunisation programme.

\section{What is the bottom line?}

- This study provides the first data from Africa, showing the effectiveness of a 2+1 PCV dosage schedule, which may inform policy regarding other immunisation programmes in low-income and middle-income country settings.

\section{Why read on?}

- The study also provides insight into the effect which different sources of controls (ie, hospital controls vs community controls) have on the $P C V$ vaccine effectiveness estimates against pneumonia.

bacteraemic pneumonia and approximately $43 \%$ of which occur in Africa. ${ }^{1}$ The WHO recommendation for immunisation of infants with pneumococcal conjugate vaccine (PCV) was based on efficacy trials undertaken in the USA and two African countries. In these randomised controlled trials, a 7-valent PCV (PCV7) in USA and 9-valent PCV in South Africa and The Gambia were efficacious in preventing vaccine-serotype invasive pneumococcal disease (75\%-95\%) and all-cause pneumonia associated with radiographically confirmed alveolar consolidation (CXR-AC; 25\%-37\%). ${ }^{2-4}$

Observational studies of the impact of PCV introduction on pneumonia from predominantly high-income countries have observed temporal declines in hospitalisation due to all-cause pneumonia ranging from $15 \%$ to $65 \%$, and $47 \%$ to $78 \%$ for healthcare provider coded 'pneumococcal pneumonia' admissions among children $<2$ years of age. $^{5} 6$ However, ecological studies may fail to identify or inadequately adjust for other factors, which may affect incidence of pneumonia 
hospitalisation. Case-control studies offer an alternate strategy for evaluating vaccine effectiveness (VE), and may also be the only method available in low-income countries where long-term administrative databases required for undertaking ecological studies are generally unavailable.

The aim of our study was to determine the effectiveness of PCV against hospitalisation for presumed bacterial pneumonia (PBP), which included CXR-AC, in HIV-uninfected South African children $\geq 8$ weeks of age.

\section{METHODS}

\section{Study setting}

We conducted a matched case-control study using two control groups from April 2010 to August 2012 in three South African public hospitals; hospital controls were enrolled at all three sites and community controls at one site. The hospitals included secondary-tertiary care-level hospital in Soweto, Johannesburg (Chris Hani-Baragwanath Academic Hospital, CHBAH) and a tertiary referral hospital in Cape Town (Red Cross War Memorial Children's Hospital) which serves an urban lowincome population; and a secondary-level care hospital in Umfolozi, KwaZulu-Natal (Ngwelezane Hospital) which services a rural, low-income population. The under-5 mortality rates in the three study settings were 27,54 and 68 per 1000 live births for Cape Town, Soweto and Umfolozi, respectively, in 2009.'

A 7-valent PCV (Prevnar-7, Wyeth Vaccines/Pfizer, Philadelphia, Pennsylvania, USA) was introduced into the South African national public immunisation programme in April 2009. From May 2011 onward, the programme transitioned to 13-valent PCV (Prevenar-13). The schedule includes two doses at ages 6 and 14 weeks and a third dose at 9 months. ${ }^{8}$ There was no catch-up campaign of older children at PCV7 introduction, while a limited catch-up campaign targeted PCV-unvaccinated children age 18-36 months between May and September 2011 following transition to PCV13. All childhood vaccines in South Africa are provided at no cost to children in the public sector. The vaccine coverage for three doses of PCV in South Africa were estimated as 64\%, 89\% and 99\% in 2010, 2011 and 2012, respectively. ${ }^{9}$

Maternal antenatal HIV prevalence in the study sites is high (Soweto 29\%, Cape Town 18\%, KwaZulu-Natal 38\% in 2009). ${ }^{10}$ Strengthening efforts to prevent mother-to-child HIV transmission from 2009 in South Africa resulted in a dramatic decline in vertical transmission of HIV infection to infants (<2\% compared to $8 \%-12 \%$ in earlier years). ${ }^{11}$

\section{Case and control enrolment \\ PBP cases}

Sequential children hospitalised with attending physician diagnosed lower respiratory tract infection (LRTI) were screened for enrolment from 07:00 to 19:00, on 5-6 days of the week. Cases admitted overnight were enrolled the following morning. Study staff was trained to enrol cases based on the inclusion criteria for the study. Vaccination status was not ascertained until after the case had been enrolled, reducing the likelihood that receipt of PCV might have influenced whether or not a case was enrolled. Cases of PBP were defined by either (1) the presence of CXR-AC or (2) any other abnormal CXR infiltrate and a quantitative C-reactive protein (CRP) of at least $40 \mathrm{mg} / \mathrm{L}$ in a child hospitalised for LRTI. ${ }^{12}$ CXRs were requested at the discretion of the attending physician and evaluated by two study physicians according to WHO guidelines for standardised interpretation of paediatric CXRs for vaccine studies. ${ }^{13}$ The readers were calibrated against the WHO standard and they were required to achieve at least $70 \%$ concordance to the WHO standard prior to involvement in this study. The decision of the third reader for discordant readings was the final reading. For a CXR to be classified as CXR-AC, both readers had to agree; discordant findings were adjudicated independently by a third study physician. For a CXR to be categorised as having any abnormal infiltrate other than CXR-AC, only one of the two primary readers were required to interpret it as such. CRP on blood samples was analysed by immunoturbidometry (717 Automated Analyzer, Boehringer Mannheim/Hitachi, Mannheim, Germany).

Cases had to be age eligible to have received $\geq 1$ PCV dose (ie, born on/after 15 February 2009). Exclusion criteria included: symptom onset $\geq 14$ days prior to admission, inability to determine HIV status, previous enrolment as PBP case, having been discharged from hospital within the past 14 days, transfer from other hospital where the duration of stay was $>1$ day or lack of documented vaccination history. The primary source of vaccination history was the child's immunisation card. Children with no documented vaccination history were not eligible to participate; except for those in which a parent/guardian reported that the child had received no vaccines other than doses given at birth, in which case the child was considered to have received zero doses of PCV.

\section{Hospital controls}

Individually matched hospital controls were matched by site, HIV status and date of birth $( \pm 1$ month from the case date of birth for cases $\leq 12$ months old, \pm 2 months for cases $>12$ months old). Children eligible for $\geq 1$ PCV dose (as for cases above) who were admitted for a non-LRTI illness that was unlikely to be due to pneumococcus (eg, exclusion of meningitis, sepsis and acute otitis media) were screened for possible participation as hospital controls. Exclusion criteria for hospital controls included: lack of documented vaccination history (as with cases, described above), hospitalisation in the past month for any suspected or proven pneumococcal disease including any LRTI, sepsis or meningitis and previous enrolment as a PBP case or hospital control. Hospital controls were targeted to be enrolled within 30 days following enrolment of the PBP cases. Hospital controls that were matched to cases that were subsequently determined to be ineligible (eg, did not meet case definition after final CXR interpretation or CRP results were available) were reallocated to other previously enrolled cases if all matching criteria were met.

\section{Community controls}

In Soweto, we also enrolled matched community controls. Approximately 23000 of the 29000 annual births in Soweto occur at $\mathrm{CHBAH}$. Potential community controls were identified using a random sampling of live births recorded on the $\mathrm{CHBAH}$ labour ward admission log on the case's date of birth. If an insufficient number of children were born on the same date, potential controls with a date of birth \pm 1 day, \pm 2 days, etc., were sought, within the same age range for matching as detailed for hospital controls. Parents of potential controls were contacted via telephone call or home visit and invited to have their children enrolled. If three attempts to contact were unsuccessful or if the child did not fulfil eligibility criteria, the next child on the random list was sought until an appropriate control was identified. All potential controls in the community were assumed to be HIV-uninfected based on the low rate $(<2 \%)$ of mother-to-child vertical transmission of HIV. ${ }^{11}$ Children were 
excluded as potential community controls if reported to be HIV-infected during the interview, or if born to a HIV-infected mother and reported to have had $>2$ hospitalisations since birth and in whom a confirmatory HIV test was unavailable or refused by the caregiver. Other exclusion criteria for community controls included those which were applied to the hospital controls. Enrolment of community controls was also targeted to occur within 30 days following case identification.

\section{SAMPLE SIZE AND POWER}

We assumed PCV effectiveness of $25 \%$ against PBP, with vaccine coverage of $85 \%$ over the course of the study and a correlation coefficient of 0.2. ${ }^{14}$ The sample size was calculated using PASS 2008 (NCSS, Kaysville, Utah, USA). We aimed to enrol sufficient cases and controls so that the analysis using each set of controls would have $\geq 80 \%$ power using a $95 \%$ significance level to measure the effectiveness in children $\geq 16$ weeks of age (eligible for $\geq 2$ PCV doses). Thus, we targeted enrolling 1450 HIV-uninfected PBP cases overall, including an estimated 950 at CHBAH. We aimed for a 2:1 ratio for enrolling hospital controls when feasible in order to increase statistical power. Three community controls were enrolled for each case in Soweto.

\section{Data collection}

Once consented, the child's medical history, demographic and household characteristics were obtained by parent interview. Vaccination histories were abstracted from parent-held vaccine records at enrolment. In addition, starting in August 2010, we photocopied vaccine records and the vaccination history was reabstracted by an independent reviewer; discrepant vaccine histories were reviewed and resolved by a third study team member. For cases and hospital controls, the medical record was also reviewed to obtain information about the current illness and HIV status.

For cases and hospital controls with no documented HIV status, testing for HIV infection was based upon initial screening with a third-generation HIV-1 ELISA test, with positive results confirmed by a different HIV-1 ELISA assay in children $\geq 18$ months of age. Qualitative HIV PCR assays were undertaken to determine HIV infection status in children $<18$ months of age who were known to have been born to an HIV-infected mother or in whom the screening HIV-ELISA test was reactive.

\section{Statistical analysis}

Only first episodes of PBP were included in this analysis. Control-children were assigned a reference date (date on which their corresponding case was hospitalised), which was used as the date for determining their vaccination status. Vaccine doses received at least 14 days before hospitalisation for cases and the reference date for controls were considered valid; all other doses were disregarded in the analysis. Children were considered to be up-to-date with PCV if they had received at least the recommended number of valid doses for their age: $\geq 1$ dose among those aged $8-15$ weeks, $\geq 2$ doses among those aged $16-40$ weeks and $\geq 3$ doses among those aged $\geq 41$ weeks.

Analyses were done using SAS software (V.9.3). We used conditional logistic regression to compare cases and controls. We estimated PCV effectiveness to be one minus the matched OR for PCV vaccination $\times 100 \%$.To assess for confounding factors, other variables were added to the model individually; variables that changed the matched OR or the $\beta$ parameter for PCV vaccination by $\geq 20 \%$ were evaluated for possible inclusion in multivariable models. For the two models using data from hospital controls (one for all sites and one for Soweto only) variables found to be confounders in either model were included in both final multivariable models. Multivariable models of adjusted vaccine effectiveness (aVE) were assessed for colinearity and two-way interactions. Crude and aVE were presented overall and by age strata based on eligibility for one, two and three doses (ie, $8-15,16-40$ and $\geq 41$ weeks); we also examined effectiveness among the age group 16-103 weeks, which represented children eligible for at least two doses and was comparable to the age range of children included in the analysis of the PCV vaccine efficacy trials. ${ }^{2-4}$ For all analyses, $\mathrm{p}$ values $<0.05$ were considered significant.

\section{Ethics}

Faculty of Health Sciences institutional review boards at the University of the Witwatersrand, University of KwaZulu-Natal, University of Cape Town and Johns Hopkins Bloomberg School of Public Health approved the study protocol. Parents/legal caregivers of all study participants provided written informed consent for their child's enrolment in the study. The trial was registered on the South African National Clinical Trial Register (DOH-27-0909-3030).

\section{RESULTS}

We enrolled 3508 children with LRTI, of whom 1705 (48.6\%) were categorised as PBP; of these, $78.0 \%$ had CXR-AC and $22.0 \%$ had other infiltrate with CRP $\geq 40 \mathrm{mg} / \mathrm{L}$ (figure 1). There were 1444 HIV-uninfected children with a first episode of PBP and adequately documented vaccination records, including 1326 (91.8\%) for whom at least one matched hospital control was enrolled (total 2075 hospital controls). Furthermore, we enrolled 2628 community controls that were matched to 889 PBP cases enrolled in Soweto (figure 1). For community controls, $74 \%$ of attempts to contact a randomly selected potential control were successful; among those contacted, $71 \%$ were found to be eligible of whom $85 \%$ were enrolled. Case-patient characteristics varied between sites in age, proportion of PBP cases with lower chest wall indrawing, proportion of cases that were mechanically ventilated, proportion of PBP cases with CXR-AC and case fatality rate (table 1 ).

Most cases (>95\%) were Black African and 58\% were males (table 2). Case and hospital controls had relatively similar characteristics, although prior hospitalisations, low birth weight and having a flush toilet were more commonly reported for cases; day care attendance and maternal education $<11$ years were more frequent among controls. Conversely, cases in Soweto differed from community controls in nearly all demographic and household characteristics examined. More than $94 \%$ of all cases and controls (hospital and community) had received their first dose of diphtheria-tetanus-pertussis Haemophilus influenzae type B vaccine. Among hospital controls, the most common discharge diagnoses were gastroenteritis $(\mathrm{n}=1015,48.9 \%)$, febrile convulsions $(\mathrm{n}=358,17.2 \%)$, underweight/malnutrition, $(\mathrm{n}=261,12.6 \%)$ and poisoning/ingestions $(\mathrm{n}=116,5.6 \%)$.

Using hospital controls, the aVE of an up-to-date PCV schedule was $20.1 \%$ (95\% CI $-9.3 \%$ to $41.6 \%)$ in children $\geq 8$ weeks of age. The point estimate of effectiveness was slightly higher in the 16-40-week age group (ie, eligible to have received at least two doses of PCV; 36.9\%) and the $\geq 41$-week group (ie, eligible to have received the complete three-dose schedule; 15.7\%) compared to the 8-15-week age group (ie, eligible for only one dose; 8.5\%), although the CIs overlapped (table 3). Among children age 16-103 weeks, the aVE of an up-to-date schedule was $39.2 \%$ (95\% CI $8.4 \%$ to $59.6 \%)$. 
3508 age-eligible children admitted with acute respiratory infection

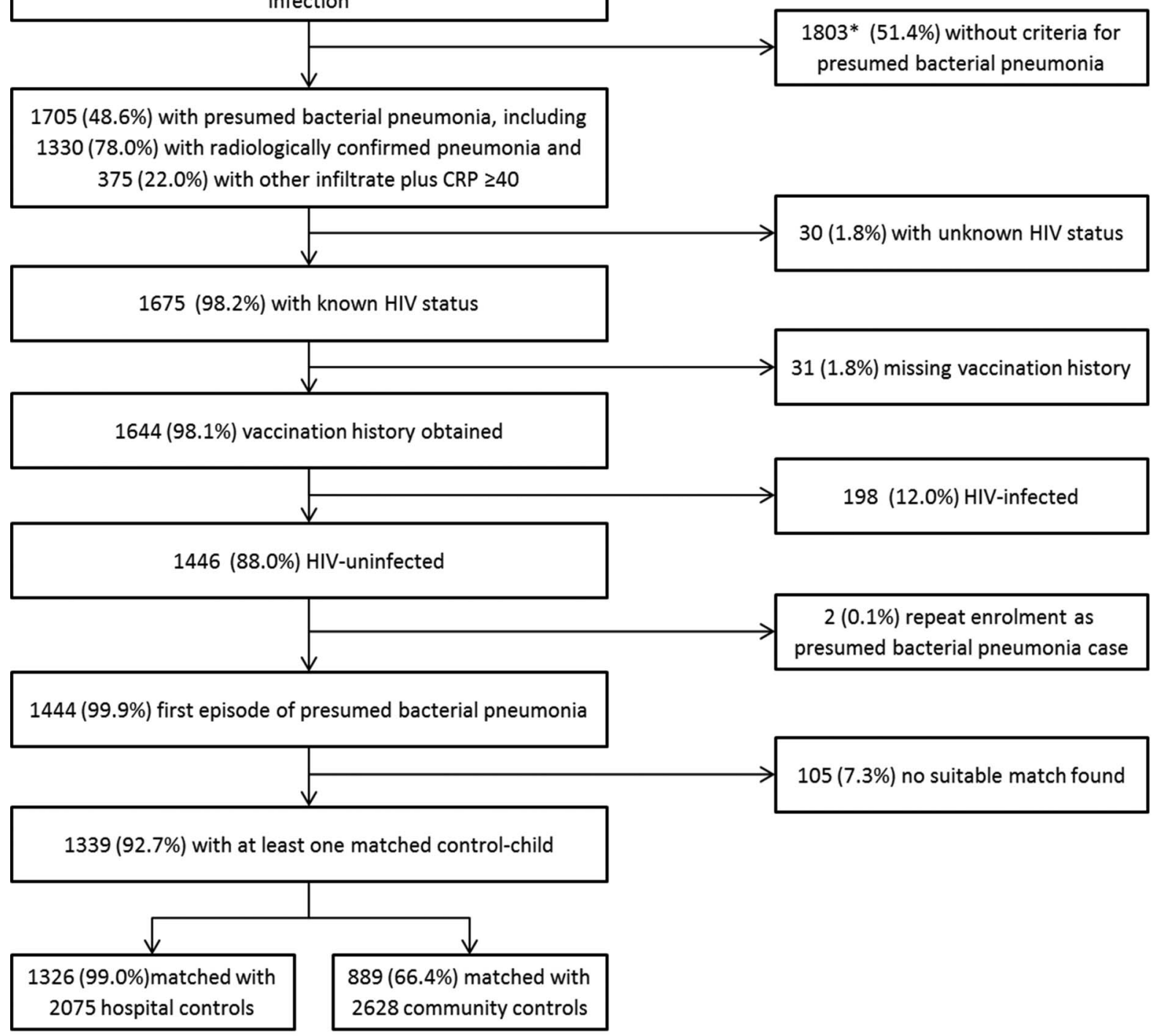

*Includes 1545 (85.7\%) children not meeting criteria for PBP and 258 (14.3\%) children insufficient data to determine whether presumed bacterial pneumonia (i.e. missing standardised interpretation of CXR or CRP)

Figure 1 Overview of enrolment of eligible cases and controls. CRP, C-reactive protein; CXR, chest radiograph; PBP, presumed bacterial pneumonia.

An exploratory analysis comparing children born before March 2011, who were mainly likely to have been vaccinated with PCV7, compared to those born thereafter and who would most likely received PCV13, did not show much difference in the aVE (see online supplementary tables 1A, B). However, the study was not powered to address the effectiveness of the specific vaccine formulations.

Using hospital controls from the Soweto site only, the aVE of an up-to-date schedule was $38.4 \%$ (95\% CI $7.7 \%$ to $58.8 \%)$ overall (ie, $\geq 8$ weeks of age) and $53.8 \%$ (95\% CI $19.5 \%$ to $73.5 \%$ ) in the $16-103$-week age group (table 3 ). Similar estimates were observed overall $(32.1 \%$; $95 \%$ CI $4.6 \%$ to $51.6 \%)$ and among the 16-103-week age group $(52.7 \%$; $95 \%$ CI $25.7 \%$ to $69.9 \%$ ) when comparing cases from Soweto with matched community controls.

For analyses using hospital controls overall and from Soweto, adjusted point estimates of effectiveness were higher than the crude estimates. However, when using community controls, adjusted models generally yielded lower or similar estimates to the unadjusted models. There was no significant change in VE when analyses were restricted to cases with CXR-AC; among $\geq 8$ weeks of age, the aVE using hospital controls was $12.2 \%$ (95\% CI $-25.7 \%$ to $38.7 \%$ ), hospital controls from Soweto only was $36.5 \%$ (95\% CI $0 \%$ to $59.7 \%$ ) and community controls was $30.6 \%$ (95\% CI $-0.7 \%$ to $52.1 \%$ ). When restricting to cases with CXR-AC among children aged 16-103 weeks, the aVE using hospital controls was $26.0 \%$ (95\% CI $-21.7 \%$ to $55.0 \%)$, hospital controls from Soweto only was 41.3\% (95\% CI $-12.3 \%$ to $69.3 \%$ ) and community controls was $50.3 \%$ (95\% CI $18.1 \%$ to $69.8 \%$ ). Similarly, excluding hospital controls that tested positive for rotavirus did not significantly alter VE estimates (data not shown). Among all cases and controls included in the analysis that had received at least one dose of PCV, the median age at receipt was 6 weeks (IQR 6-7 weeks); for the second PCV dose, the median age was 15 weeks (IQR 14-17 weeks); and for the third dose, the median age was 
Table 1 Demographic and clinical characteristics of presumed bacterial pneumonia cases*

\begin{tabular}{|c|c|c|c|c|c|}
\hline \multirow[b]{2}{*}{ Case characteristics } & \multicolumn{4}{|c|}{ Cases with hospital controls } & \multirow{2}{*}{$\begin{array}{l}\text { Cases with } \\
\text { community controls } \\
\text { Soweto } \\
\mathrm{n}=889 \\
\mathrm{n}(\%)\end{array}$} \\
\hline & $\begin{array}{l}\text { All sites } \\
n=1326 \\
n(\%)\end{array}$ & $\begin{array}{l}\text { Soweto } \\
n=893 \\
n(\%)\end{array}$ & $\begin{array}{l}\text { Cape Town } \\
\mathrm{n}=274 \\
\mathrm{n}(\%)\end{array}$ & $\begin{array}{l}\text { KwaZulu-Natal } \\
n=159 \\
n(\%)\end{array}$ & \\
\hline \multicolumn{6}{|l|}{ Age of admission (weeks) } \\
\hline Median & 35.0 & 38.0 & 30.0 & 25.0 & 38.0 \\
\hline Mean & 43.1 & 46.4 & 36.0 & 36.3 & 46.0 \\
\hline Range & $8-167$ & $8-167$ & 8-119 & $8-154$ & $8-167$ \\
\hline CXR-AC & $1040(78.4)$ & $742(83.1)$ & $168(61.3)$ & $130(81.8)$ & $741(83.4)$ \\
\hline$C R P \geq 40+C X R-0 I$ & $286(21.6)$ & $151(16.9)$ & $106(38.7)$ & $26(17.2)$ & $148(16.6)$ \\
\hline Lower chest indrawing & $666(52.9)$ & $274(32.6)$ & $235(89.5)$ & $157(100.0)$ & $273(32.6)$ \\
\hline Wheezing & $603(47.6)$ & $387(45.3)$ & $153(59.8)$ & $63(40.1)$ & $381(44.8)$ \\
\hline Mechanical ventilation & $34(2.7)$ & $6(0.7)$ & $6(2.2)$ & $22(14.3)$ & $6(0.7)$ \\
\hline Death & $13(1.0)$ & $2(0.2)$ & $1(0.4)$ & $10(6.3)$ & $2(0.2)$ \\
\hline
\end{tabular}

Table 2 Characteristics of all PBP cases with hospital controls, cases with hospital controls from Soweto only and cases with community controls

\begin{tabular}{|c|c|c|c|c|c|c|c|c|c|}
\hline \multirow[b]{3}{*}{ Characteristic } & \multicolumn{3}{|c|}{ Cases with hospital controls } & \multicolumn{3}{|c|}{$\begin{array}{l}\text { Cases with hospital controls, Soweto } \\
\text { only }\end{array}$} & \multicolumn{3}{|c|}{ Cases with community controls } \\
\hline & $\begin{array}{l}\text { Cases } \\
n=1326\end{array}$ & $\begin{array}{l}\text { Controls } \\
\mathrm{n}=2075\end{array}$ & & $\begin{array}{l}\text { Cases } \\
\mathrm{n}=893\end{array}$ & $\begin{array}{l}\text { Controls } \\
n=1453\end{array}$ & & $\begin{array}{l}\text { Cases } \\
\mathrm{n}=889\end{array}$ & $\begin{array}{l}\text { Controls } \\
n=2628\end{array}$ & \\
\hline & n (\%) & n (\%) & $\begin{array}{l}\text { Matched } \\
p \text { value }\end{array}$ & n (\%) & n (\%) & $\begin{array}{l}\text { Matched } \\
p \text { value }\end{array}$ & n (\%) & n (\%) & $\begin{array}{l}\text { Matched } \\
\mathrm{p} \text { value }\end{array}$ \\
\hline Black race & $1266(95.5)$ & $1979(95.4)$ & 0.062 & $883(98.9)$ & $1426(98.1)$ & 0.149 & $879(98.9)$ & $2564(97.6)$ & 0.028 \\
\hline Male sex & $765(57.7)$ & $1191(57.4)$ & 0.824 & $525(58.8)$ & $832(57.3)$ & 0.604 & $521(58.6)$ & $1343(51.2)$ & $<0.001$ \\
\hline Weight-for-age Z score $<2$ & $332(25.1)$ & $558(26.9)$ & 0.524 & $225(25.2)$ & $419(28.9)$ & 0.096 & $224(25.2)$ & $145(5.5)$ & $<0.001$ \\
\hline Any chronic condition* & $41(3.1)$ & $85(4.1)$ & 0.277 & $17(1.9)$ & $44(3.0)$ & 0.147 & $17(1.9)$ & $17(0.6)$ & 0.002 \\
\hline Prior hospitalisation & $265(20.0)$ & $295(14.2)$ & $<0.001$ & $155(17.4)$ & $185(12.8)$ & 0.003 & $151(17.0)$ & $228(8.7)$ & $<0.001$ \\
\hline $\begin{array}{l}\text { Prior pneumonia } \\
\text { hospitalisation }\end{array}$ & $144(11.1)$ & $100(4.8)$ & $<0.001$ & $86(9.9)$ & $65(4.5)$ & $<0.001$ & $83(9.6)$ & $99(3.8)$ & $<0.001$ \\
\hline Low birth weight† & $296(22.7)$ & $326(15.8)$ & $<0.001$ & $191(21.8)$ & $223(15.4)$ & $<0.001$ & $187(21.5)$ & $386(14.7)$ & $<0.001$ \\
\hline Mother HIV-infected & $432(33.3)$ & $620(30.4)$ & 0.094 & $288(32.8)$ & $428(30.1)$ & 0.303 & $286(32.6)$ & $601(22.9)$ & $<0.001$ \\
\hline TB contact & $112(8.5)$ & $165(8.0)$ & 0.524 & $53(6.0)$ & $75(5.2)$ & 0.353 & $53(6.0)$ & $143(5.5)$ & 0.632 \\
\hline $\begin{array}{l}\text { Exclusive breast fed } \\
<4 \text { months }\end{array}$ & $698(52.8)$ & $1102(53.2)$ & 0.939 & $464(52.2)$ & $770(53.1)$ & 0.721 & $464(52.4)$ & $1635(62.2)$ & $<0.001$ \\
\hline Brick house & $870(66.1)$ & $1355(65.5)$ & 0.198 & $615(69.6)$ & $973(67.2)$ & 0.244 & $612(69.6)$ & $2064(78.5)$ & $<0.001$ \\
\hline Indoor water supply & $474(35.8)$ & $699(33.8)$ & 0.664 & $309(34.6)$ & $517(35.7)$ & 0.688 & $302(34.0)$ & $1234(47.0)$ & $<0.001$ \\
\hline Flush toilet & $1020(77.0)$ & $1479(71.4)$ & 0.012 & $770(86.3)$ & $1194(82.3)$ & 0.010 & $763(85.9)$ & $2339(89.0)$ & 0.011 \\
\hline Coal/wood primary fuel & $47(3.6)$ & $103(5.0)$ & 0.797 & $0(0)$ & $2(0.1)$ & 0.973 & $0(0)$ & $6(0.2)$ & 0.973 \\
\hline Primary care giver smokes & $86(6.5)$ & $117(5.6)$ & 0.615 & $48(5.4)$ & $89(6.1)$ & 0.447 & $47(5.3)$ & $29(1.1)$ & $<0.001$ \\
\hline Day care & $216(16.4)$ & $436(21.0)$ & $<0.001$ & $165(18.5)$ & $330(22.8)$ & 0.024 & $156(17.6)$ & $515(19.6)$ & 0.091 \\
\hline Mother education $<11$ & $386(30.0)$ & $686(33.2)$ & 0.021 & $214(24.8)$ & $433(29.9)$ & 0.005 & $211(24.5)$ & $543(20.7)$ & 0.016 \\
\hline Crowding $\ddagger$ & $685(51.8)$ & $1092(52.7)$ & 0.654 & $443(49.8)$ & $794(54.8)$ & 0.013 & $443(50.0)$ & $814(31.0)$ & $<0.001$ \\
\hline At least one dose of DTP & $1258(94.9)$ & $1954(94.1)$ & 0.231 & $855(95.7)$ & 1371 (94.4) & 0.111 & $851(95.7)$ & $2527(96.2)$ & 0.494 \\
\hline \multicolumn{10}{|l|}{ PCV doses§ } \\
\hline 0 & $144(10.9)$ & $234(11.3)$ & - & $75(8.4)$ & $133(9.2)$ & - & $76(8.6)$ & $157(6.0)$ & - \\
\hline 1 & $394(29.7)$ & $579(27.9)$ & 0.318 & $229(25.6)$ & $368(25.3)$ & 0.674 & $234(26.3)$ & $614(23.4)$ & 0.191 \\
\hline 2 & $476(35.9)$ & $729(35.1)$ & 0.961 & $340(38.1)$ & $519(35.7)$ & 0.978 & $338(38.0)$ & $1066(40.6)$ & $<0.001$ \\
\hline 3 & $296(22.3)$ & $498(24.0)$ & 0.325 & $237(26.5)$ & $401(27.6)$ & 0.371 & $232(26.1)$ & $755(28.7)$ & $<0.001$ \\
\hline 4 & $16(1.2)$ & $35(1.7)$ & 0.173 & $12(1.3)$ & $32(2.2)$ & 0.052 & $9(1.0)$ & $36(1.4)$ & 0.004 \\
\hline
\end{tabular}




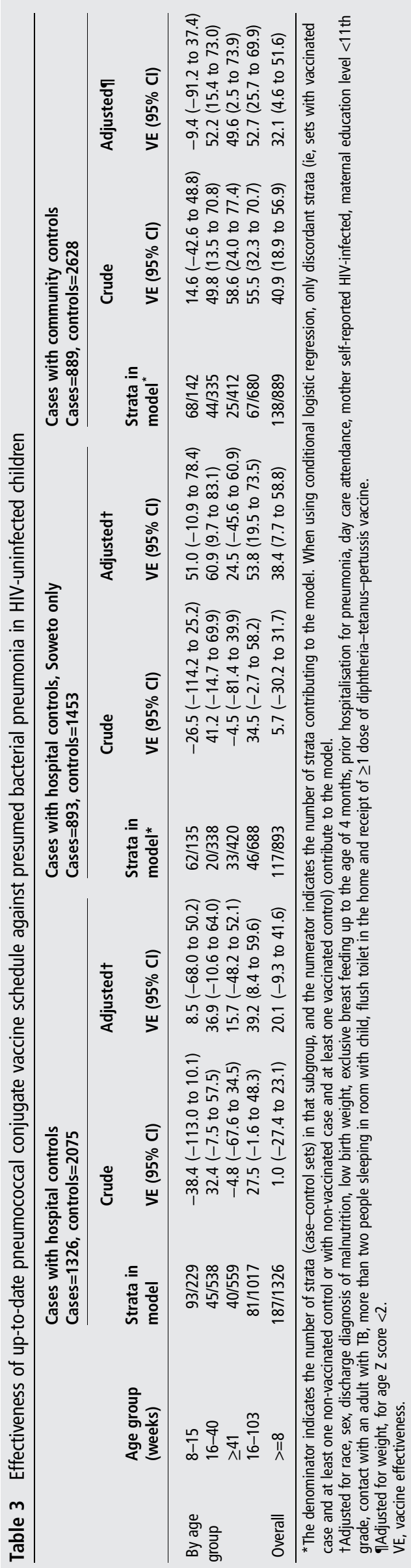

39 weeks (IQR 39-41 weeks). Although we intended to evaluate PCV effectiveness in both HIV-infected and -uninfected children, we were unable to enrol sufficient numbers of cases to evaluate VE among HIV-infected children.

\section{DISCUSSION}

This is the first African study to demonstrate effectiveness of PCV in prevention of PBP hospitalisation, when used as part of routine childhood immunisation with a novel vaccination schedule at 6,14 and 40 weeks of age. The data corroborate those of earlier randomised controlled phase III trials of 7-11 valent PCVs. ${ }^{2-4} 1215$ The observed protection among children age $16-103$ weeks (39.2\%) is similar to the $33 \%$ efficacy against CXR-AC observed in a meta-analysis of PCV in the randomised, placebo-controlled trials in which follow-up time was largely limited to children $<2$ years of age. ${ }^{5}$ Also, the overall adjusted VE of $20.1 \%$ for children who were up to date for vaccination and $\geq 8$ weeks of age was similar to the vaccine efficacy of $25 \%$ (95\% CI $4 \%$ to $41 \%$ ) observed in a randomised, placebocontrolled trial of a 9-valent PCV against CXR-AC previously undertaken in Soweto, South Africa. ${ }^{16}$ Notably, the national PCV schedule implemented in South Africa and evaluated in this study differed from those in the randomised controlled trials where children received three doses within 4-6 months of age with or without a booster in the second year of life. As such, the observation of similar VE among children age 16-40 weeks, a group eligible to have received only two doses of PCV in the current study, is important, particularly since young infants are at greatest risk of pneumonia-associated morbidity and mortality. ${ }^{1}$

Additional factors must be considered in comparing our results to those of PCV clinical trials. First, the results although heavily weighted in regard to evaluating PCV7 effectiveness, are a composite for VE following the transition from PCV7 to PCV13 during the course of the study. That some children received PCV13, which includes serotypes 1 and 5, may explain in part why our results were similar to those seen in the clinical trials that used the 9-valent $\mathrm{PCV}^{16}$ In addition, our case definition (PBP) was broader than that proposed by WHO (ie, CXR-AC) for measuring PCV VE against pneumonia, although VE estimates in our study remained similar when analysis was restricted to only cases with CXR-AC. The decision to use an expanded case definition of PBP was based on a previous post-hoc analysis that this composite endpoint provided greater sensitivity for detecting the burden of pneumonia prevented by $\mathrm{PCV}^{17}{ }^{18}$ without affecting the specificity of the pneumonia endpoint for establishing vaccine efficacy. One randomised controlled trial of a 10-valent PCV that used the same case definition as ours reported an efficacy of $26 \%(95 \%$ CI $8 \%$ to $40 \%)$ against radiologic pneumonia. ${ }^{12}$

Conducting case-control studies in a way that minimises bias, and therefore provides accurate results, can be challenging. This includes selection of appropriate controls that represent the same source population of cases and adjustment for important confounders is crucial. In this study, we used two different control groups. The hospital controls had similar characteristics as the case-patients, yet the analysis using that control group was heavily affected by confounding. Community controls, not unexpectedly, differed in many characteristics from cases, particularly in relation to known risk factors for pneumonia such as male gender, malnutrition, chronic underlying conditions, low birth weight, absence of exclusive breast feeding at age $<4$ months, overcrowding and maternal HIV status. Nevertheless, VE estimates using this control group were less 
affected by other variables and results of adjusted analyses using each control group were remarkably consistent. Of note, in Soweto, there is readily available access to healthcare at little direct cost to families. Hospital controls in other settings with barriers to accessing healthcare may be more problematic. Our results highlight the importance of adjusting for confounding in the analysis. However not all confounders can be easily or accurately measured, and it is possible that key factors were not adjusted for in the analysis. A further limitation of our study related to the reliability of verbally reporting vaccination status, in which accepted parental declaration of non-vaccination (beyond birth doses) was viewed as evidence of zero PCV doses. This was done, as children who have not received any vaccines beyond birth doses are unlikely to have a card documenting their lack of vaccination, however excluding this group of children could lead to important bias. Therefore we chose to include children whose parents reported no vaccines received beyond those given at birth.

Other limitations of our study include having restricted community control enrolment to only one of the three sites. We observed important differences in the severity of illness among cases across sites, with a case fatality proportion of $6.3 \%$ in KwaZulu-Natal and $<1 \%$ in Cape Town and Soweto; such variability may reflect differences in access to care, which can influence the optimal control group. However, due to logistical and financial challenges of undertaking enrolment of community controls at the other sites, we were only able to enrol community controls in Soweto. Although we included children from three diverse settings, the findings may not be generalisable to all contexts in South Africa or to other African countries. Furthermore, although we aimed at limiting hospital controls to illnesses in which pneumococcus was unlikely to have been the aetiological agents, the inclusion of children with febrile convulsions and other non-specified febrile illness as hospital controls, could inadvertently have included children with pneumococcal illness as hospital controls, potentially biasing towards the null.

In conclusion, our study corroborates the findings from randomised controlled trials and ecological studies from highincome countries, which establish a major role of PCV in protecting young children against pneumonia. We present the first estimates of PCV protection against a pneumonia endpoint using a case-control methodology. Our data support the use of a two-dose primary schedule plus a booster dose and provide evidence of PCV effectiveness against pneumonia in the context of a routine immunisation programme in an African country.

\section{Author affiliations \\ ${ }^{1}$ Medical Research Council: Respiratory \& Meningeal Pathogens Research Unit, University of the Witwatersrand, Johannesburg, South Africa \\ ${ }^{2}$ Department of Science and Technology/National Research Foundation, Vaccine Preventable Diseases, University of the Witwatersrand, Johannesburg, South Africa ${ }^{3}$ National Institute for Communicable Diseases, Division of National Health Laboratory Service, Center for Vaccines and Immunology, Johannesburg, South Africa ${ }^{4}$ Department of Paediatrics and Child Health, Red Cross War Memorial Hospital, University of Cape Town, Cape Town, South Africa \\ ${ }^{5}$ Ngwelezane Hospital, University of KwaZulu-Natal, KwaZulu Natal, South Africa \\ ${ }^{6}$ Centers for Diseases Control and Prevention, Atlanta, Georgia, USA}

Contributors Conception and design of the study: SAM, MJG, CGW, JRV. Acquisition of data: MJG, HJZ, CNK, CM, SN, DPM. Analysis of data: JRV, ERZ. Interpretation of data: SAM, MJG, HJZ, CNK, ERZ, CGW, JRV. Drafting of article: SAM. Critically revising drafts of article: MJG, HJZ, CNK, CM, SN, DPM, ERZ, CGW, JRV. Final approval of submitted version: SAM, MJG, HJZ, CNK, CM, SN, DPM, ERZ, CGW, JRV.
Funding Funding for the study was provided by the Accelerated Vaccine Introduction (Special Studies) of the GAVI Alliance (grant number: GAV1144-08410(RT). The study sponsor had no role in the design or implementation of the study, analysis or reporting of the results. The corresponding author had full access to all the data in the study and had final responsibility for the decision to submit for publication. The findings and conclusions in this report are those of the authors and do not necessarily represent the official position of GAVI or the Centers for Disease Control and Prevention.

Competing interests SAM reports a grant from GAVI Alliance, during the conduct of the study; grants and personal fees from GSK, grants and personal fees from Pfizer, grants from Novartis, grants and personal fees from Sanofi Pasteur, outside the submitted work. MJG reports a grant from GAVI Alliance, during the conduct of the study; personal fees from GlaxoSmithKline, personal fees from Sanofi Pasteur, outside the submitted work.

Ethics approval University of the Witwatersrand, University of KwaZulu-Natal, University of Cape Town and Johns Hopkins Bloomberg School of Public Health approved the study protocol.

Provenance and peer review Not commissioned; externally peer reviewed.

Data sharing statement Unpublished data is available to the authors only.

\section{REFERENCES}

1 Walker CL, Rudan I, Liu L, et al. Global burden of childhood pneumonia and diarrhoea. Lancet 2013:381:1405-16.

2 Klugman KP, Madhi SA, Huebner RE, et al. A trial of a 9-valent pneumococcal conjugate vaccine in children with and those without HIV infection. N Engl J Med 2003;349:1341-8.

3 Cutts FT, Zaman SM, Enwere G, et al. Efficacy of nine-valent pneumococcal conjugate vaccine against pneumonia and invasive pneumococcal disease in The Gambia: randomised, double-blind, placebo-controlled trial. Lancet 2005;365:1139-46.

4 Hansen J, Black S, Shinefield $H$, et al. Effectiveness of heptavalent pneumococcal conjugate vaccine in children younger than 5 years of age for prevention of pneumonia: updated analysis using World Health Organization standardized interpretation of chest radiographs. Pediatr Infect Dis J 2006;25:779-81.

5 Fitzwater SP, Chandran A, Santosham M, et al. The worldwide impact of the seven-valent pneumococcal conjugate vaccine. Pediat Infect Dis J 2012;31:501-8.

6 Loo JD, Conklin L, Fleming-Dutra KE, et al. Systematic review of the effect of pneumococcal conjugate vaccine dosing schedules on prevention of pneumonia. Pediatr Infect Dis J 2014;33(Suppl 2):S140-51.

7 CoMMIC. Interim report of the Committee on Morbidity and Mortality in Children under 5 years (CoMMiC):2012. 2012. http://wwwdohgovza\%2Fdocs\%2Freports\% 2F2013\%2Fmorbidity_\%26_mortalitypdf

8 Jones SA, Groome M, Koen A, et al. Immunogenicity of seven-valent pneumococcal conjugate vaccine administered at 6, 14 and 40 weeks of age in South African infants. PLOS ONE 2013;8:e72794.

9 Madhi SA, Bamford L, Ngcobo N. Effectiveness of pneumococcal conjugate vaccine and rotavirus vaccine introduction into the South African public immunisation programme. S Afr Med J 2014;104(3 Suppl 1):228-34.

10 Department of Health. http://www.health-e.org.za/2012/12/11/2011-nationalantenatal-hiv-syphilis-prevalence-survey-released/ (accessed 30 Jul 2014).

11 Barron P, Pillay Y, Doherty T, et al. Eliminating mother-to-child HIV transmission in South Africa. Bull World Health Organ 2013;91:70-4.

12 Tregnaghi MW, Saez-Llorens $X$, Lopez $P$, et al. Efficacy of pneumococcal nontypable Haemophilus influenzae protein D conjugate vaccine (PHiD-CV) in young Latin American children: a double-blind randomized controlled trial. PLOS Med 2014;11: e1001657

13 Cherian T, Mulholland EK, Carlin JB, et al. Standardized interpretation of paediatric chest radiographs for the diagnosis of pneumonia in epidemiological studies. Bull World Health Organ 2005;83:353-9.

14 Dupont WD. Power calculations for matched case-control studies. Biometrics 1988:44:1157-68.

15 Lucero MG, Nohynek $H$, Williams $G$, et al. Efficacy of an 11-valent pneumococcal conjugate vaccine against radiologically confirmed pneumonia among children less than 2 years of age in the Philippines: a randomized, double-blind, placebo-controlled trial. Pediatr Infect Dis J 2009:28:455-62.

16 Madhi SA, Kuwanda L, Cutland C, et al. The impact of a 9-valent pneumococcal conjugate vaccine on the public health burden of pneumonia in HIV-infected and -uninfected children. Clin Infect Dis 2005;40:1511-18.

17 Madhi SA, Klugman KP. World Health Organisation definition of "radiologically-confirmed pneumonia" may under-estimate the true public health value of conjugate pneumococcal vaccines. Vaccine 2007;25:2413-19.

18 Madhi SA, Kohler M, Kuwanda L, et al. Usefulness of C-reactive protein to define pneumococcal conjugate vaccine efficacy in the prevention of pneumonia. Pediatr Infect Dis J 2006;25:30-6. 